\title{
Update on the Evidence for Functional Rhinoplasty Techniques
}

David Chan, MD ${ }^{1}$ and Taha Z. Shipchandler, MD, FACS ${ }^{1}$

1. Division of Facial Plastic \& Reconstructive Surgery, Department of Otolaryngology,, Indiana University School of Medicine, Indianapolis, IN

Corresponding Author: Taha Z. Shipchandler, MD, FACS

Director, Facial Plastic \& Reconstructive Surgery

Residency Program Director

Indiana University School of Medicine

tshipcha@iupui.edu

(317)-948-FACE

(317)-688-4864

This is the author's manuscript of the article published in final edited form as:

Chan, D., \& Shipchandler, T. Z. (2015). Update on the evidence for functional rhinoplasty techniques. Current Opinion in Otolaryngology \& Head and Neck Surgery, 23(4), 265-271.

http://doi.org/10.1097/MOO.0000000000000172 


\section{Purpose of review}

Functional rhinoplasty is a term that is commonly used to describe any technique used to address nasal obstruction in the nasal valve region. Etiology of nasal valve collapse differs based on the site. Internal valve collapse may be idiopathic or associated with previous rhinoplasty, trauma, or weakened cartilage in older patients. External valve collapse is usually idiopathic and less likely to be associated with previous surgery. Various techniques have been developed over time, and the data in support of functional rhinoplasty continues to increase.

\section{Recent findings}

This article discusses popular techniques currently in use as well as newer techniques that have been described over the past several years. In addition, there is a trend toward utilizing objective testing and validated assessment scales to assess post-operative changes.

\section{Summary}

Functional rhinoplasty utilizes a multitude of techniques to address nasal valve collapse. While high-level data is lacking, the current literature is in support of nasal valve correction to improve nasal breathing. Most importantly, the technique employed is unique for each patient.

\section{Keywords}

Functional rhinoplasty, Nasal Valve Collapse, Nasal Airway Obstruction 


\section{Introduction}

Nasal airway obstruction (NAO) is a common complaint that presents to physician offices. It is estimated that roughly 5 billion dollars are spent on medical treatments with an additional 60 million dollars spent on surgical interventions annually.[1] Causes of NAO can be inflammatory or anatomic. Inflammatory causes include allergic rhinitis, nasal polyps, and chronic sinusitis. Anatomic causes include septal deviation, turbinate hypertrophy, and nasal valve collapse (NVC). Of the anatomic causes, NVC is a common reason for NAO. It is estimated that $13 \%$ of the general population suffer from NVC.[2] Many of these patients will have already failed to find relief with nasal steroids, decongestants, and nasal tape strips. They are then referred to an Otolaryngologist or Facial Plastic surgeon for a surgical evaluation.

Functional rhinoplasty is a term that is commonly used to describe any technique used to address nasal obstruction in the nasal valve region. Etiology of nasal valve collapse differs based on the site. Internal valve collapse may be idiopathic or associated with previous rhinoplasty, trauma, or in older patients with weakened cartilages. External valve collapse is usually idiopathic and less likely to be associated with previous surgery.[3]

\section{Anatomy and Physiology}

Proper diagnosis of patients with NVC first requires an understanding of the anatomy and physiology of the nasal valve area. Mink is credited as the first to use the term 'nasal valve' in 1903 and the definition has been modified over the years. The nasal valve is the narrowest part of the airway and, therefore, contributes the most resistance to airflow. It is divided into external and internal parts. The external nasal valve is the area formed by the caudal septum, medial crura of the alar cartilages, alar rim, and nasal sill. The internal nasal valve is the area bounded by the 
caudal edge of the upper lateral cartilage (ULC), nasal septum, anterior head of the inferior turbinate, and laterally by the upper lateral cartilage. The internal nasal angle is the area between the upper lateral cartilage and septum, which is normally 10 to 15 degrees in Caucasians and slightly wider in African Americans and Asians.[4-7] The nasal valve area is recognized as the area of highest resistance in the airway. Obstruction at the valve can be either static or dynamic and could lead to increased resistance to airflow. Static obstructions can be due to septal deviation, turbinate hypertrophy, inherent concavity of the nasal cartilages, or avulsion of the attachment of the ULC to the septum. Dynamic obstruction occurs when the alar rim or sidewall collapses during inspiration, which is usually due to weakened cartilage or dilator muscles.[2] Dynamic collapse of the nasal sidewall occurs according to Bernoulli’s principle as airflow through the nose causes a vacuum effect resulting in collapse.[8]

\section{Diagnosis}

A panel convened by the American Academy of Otolaryngology - Head and Neck Surgery (AAO-HNS) agreed that NVC is best diagnosed by history and physical exam. The key characteristic reported by patients with NVC is decreased nasal airflow. Sleep can be adversely affected by NVC. A history of nasal surgery should be obtained.[6] History of trauma and medical treatment can be helpful as well. Poirrier et al[9] recently published data on a grading system validated for external valve collapse. A score of 0 - 2 is given per side based on the extent

of external valve collapse. Using a standardized grading system may help add consistency to reporting and with standardizing future studies. 
Nasal valve collapse can be either static or dynamic. Static causes are due to narrow areas of the internal and external nasal valves. Dynamic collapse occurs when the nasal sidewall collapses during inspiration, which is due to a lack of resiliency of the cartilages. Identification of lateral nasal wall or alar rim collapse on inspiration is consistent with NVC. Clinicians use both the Cottle and modified Cottle maneuvers to diagnose NVC.[10] Attention should also be paid to hypertrophic turbinates, caudal septal deflections, and wide medial crural footplates. Anterior rhinoscopy should be routine in the examination to evaluate the nasal valve area. Although the panel did not recommend routine use of a nasal endoscope during the nasal exam, the authors do advocate its use.[6, 7] Proponents report that the nasal valve area can be better visualized with an endoscope without manipulating the lateral nasal wall and caudal ULC. In addition, Lanfranchi et al. reported that up to 30\% of rhinoplasty patients had other nasal pathologies that required surgical attention to improve nasal breathing.[11]

While various objective tests, such as rhinomanometry, nasal peak inspiratory flow, and acoustic rhinometry, are available to the clinician to assess nasal obstruction[12], they have not been shown to strongly correlate with patient complaints. $[6,13-16]$ Ishii et al. reviewed the literature for evidence supporting the use of diagnostic tests but ultimately found that history and physical exam are the clinician's best tools in diagnosing NVC.[17] More recently, however, Tsounis et al showed that peak nasal inspiratory flow (PNIF) correlated well with patient complaints of nasal obstruction.[18] In addition, Menger et al not only showed correlation between patient symptoms and PNIF, but also that post-operative improvement was noted as well.[19] Palesy et al also found a positive correlation between patient-outcome measuring devices with improvement in NPIF scores.[16] Validated disease-specific quality-of-life instruments, such as the Nasal Obstruction Symptom Evaluation (NOSE) scale and Visual 
Analog Scale (VAS), have been helpful in assessing outcomes and are now commonly used in research and clinical practice.[16, 20-23] A small number of studies have looked at the utility of computed tomography in examining the nasal airway with inconsistent results.[24-26]

\section{Treatment Options}

There exist a myriad of methods in the treatment of both internal and external valve collapse. As our understanding of the pathologic process deepens, new techniques and/or modifications of old techniques have been used. The following are descriptions of popular techniques utilized in the treatment of nasal valve collapse.

\section{Alar Batten Grafts}

First introduced by Tardy et al[27], alar batten grafts are curvilinear grafts that are used to correct both internal and external valve collapse depending on the anatomic placement of the grafts in the nose. Materials used for battens grafts include auricular, septal (Figures $1 \& 2$ ), and rib cartilage and allografts.[28, 29] Precise placement of the grafts are well described by Toriumi et al[3] and Chua and Park.[30] Batten grafts are used to strengthen support of the lateral nasal wall and prevent collapse. Cervelli et al[31] reported that all patients in his study had improvement in nasal breathing for both internal and external valve collapse. In two separate studies, Sufyan and Shipchandler et al[29, 32] noted improvement in nasal breathing with batten grafts and also decreased usage of nasal steroids. The latter possibly due to less need for steroids as a result of larger nasal passageways. One noted disadvantage of the batten graft is fullness at the graft site and possible visibility of the graft contour in individuals with thin nasal $\operatorname{skin}[3,33$, 34]. 


\section{Spreader Grafts}

Spreader grafts were first introduced by Sheen[35] to reconstruct the internal nasal valve and midvault (Figure 3). The grafts are crafted from septal or auricular cartilage or alloplastic implants[36] and placed between the dorsal septum and upper lateral cartilage. The grafts can be placed via an open or endonasal approach.[37, 38] Spreader grafts are recognized as the gold standard in reconstruction and restoration of the middle vault.[37, 39] In addition, spreader grafts have a role in improving breathing in internal valve collapse by widening the middle vault and repositioning the upper lateral cartilages.[3, 20, 40] Interestingly, despite the subjective improvement reported by patients, objective measures have not show a dramatic increase in nasal airflow.[41, 42]

\section{Spreader Flaps}

The spreader flap technique was first reported in the late 1990s to widen the midvault.[43] Instead of utilizing septal cartilage for the spreader graft, the upper lateral cartilage is folded inward and sutured to the dorsal septum thus widening the nasal valve angle. Yoo and Most examined the use of this technique in preventing post-rhinoplasty nasal obstruction.[44] In a prospective fashion, they utilized the NOSE survey to evaluate patients preand post-operatively. In patients who had nasal airway obstruction, the spreader flap technique significantly improved nasal breathing as evidenced by a reduction in the NOSE score. Saedi et al[39] found that spreader flaps did preserve the midvault, but did little to augment the nasal cross-sectional area. While some authors have reported encouraging results over the years, more studies are needed to further define the role of spreader flaps in nasal valve collapse. 


\section{Flare Sutures}

Introduced by Park[45], flaring sutures were designed to address the nasal valve angle (Figure 4). Spreader grafts were effective in widening the middle vault but was not believed to have much of an effect on the internal nasal valve angle. Batten grafts were useful in reinforcing the nasal sidewall to prevent lateral wall collapse. Park recognized that neither method addressed the nasal valve angle thus the flare suture was described. The sutures are placed in the upper lateral cartilages in a horizontal mattress fashion such that when tightened over the nasal dorsum, the cartilages “flare outward.” Park’s initial report demonstrated sustained improvement 1 year after surgery. Schlosser and Park[46] objectively showed that flaring sutures and flaring sutures plus spreader grafts improved nasal cross-sectional areas more so than spreader grafts alone. Shadfar et al[42] examined the changes in nasal airflow between various flare suture placements versus spreaders grafts using a computational fluid dynamics model. They found that medially placed flare sutures improved airflow and reduced resistance more so than spreaders grafts alone or spreader grafts plus flare sutures. Flare sutures appear to be an effective tool in the treatment of nasal valve collapse either in isolation or in conjunction with other grafting techniques.

\section{Alar Rim Grafts}

First described by Troell et al, the alar rim graft was used to correct external nasal valve collapse (Figure 5). Long, thin grafts carved from septal cartilage are placed into precisely made tunnels along the alar margin just caudal to the border of the lateral crus.[47] Guyuron and Rohrich et al, used the alar rim graft to correct notching of the alar rim.[48, 49] Boahene and Hilger used alar rim grafts mainly to correct cephalic rotation of the LLC and alar flaring, but it 
has also been used for dynamic external valve collapse.[50] In their cohort of 31 patients, there were no graft infections, displacements, or extrusions at 6 month follow up. They also noted that in those patients who had dynamic collapse at the alar margin, there was notable improvement in alar support with less collapse on deep inspiration.

\section{Butterfly Graft}

Initially described by Clark et al, the conchal cartilage butterfly graft was used to repair nasal valve collapse after rhinoplasty.[51] The graft is placed over the scroll area and acts to widen the nasal valve angle (Figures 6 and 7).[5] Clark et al reported that all of their subjects experienced subjective improvement in nasal breathing and only 2 reported that the results made their nose look worse. In a follow up study by Friedman et al, they examined the usefulness of the butterfly graft in primary functional rhinoplasty.[52] They found that a majority of patients (90\%) were satisfied by the improvement in their nasal breathing, but only $88 \%$ were satisfied with the cosmetic appearance. Dissatisfaction included nasal dorsal or supra-trip irregularities and nasal sidewall fullness. Stacey et al compared spreader grafts to butterfly grafts and found that both improved nasal breathing without significant differences but the butterfly graft did do better in terms of improving snoring, sleep, and continuous positive airway pressure use in patients with obstructive sleep apnea.[53] Given that between 3\% - 19\% of patients reported dissatisfaction with the cosmetic result of the butterfly graft technique, Chaiet et al examined changes to the nasal tip based on pre- and post-operative photos. They found the tip on frontal view can have a change in width between $-10.2 \%$ to $15.7 \%$, and tip projection can change between $-23.4 \%$ to $+15.0 \%$. They acknowledged that patients with thin skin may not adequately hide the graft, and that proper counseling is needed if this technique is utilized.[54] 


\section{Radiofrequency and Bone Anchored Suspension}

Radiofrequency-induced thermotherapy (RFITT) is often used for inferior turbinate reduction, however, Seren examined its use in patients with dynamic internal valve collapse.[55] The RFITT probe is inserted into a mucosal pocket along the lateral crura towards the piriform aperture. Three sites are ablated for 10 seconds each delivering a total of $100 \mathrm{~J}$. Scar tissue forms in that location and causes tissue contraction and increased tone of the nasal musculature. The increased tone would in theory reduce collapse of the lateral nasal wall. Seren followed 28 patients for 16 weeks and using the VAS, he showed a significant reduction in scores after treatment. Weissman and Most further investigated RFITT compared to bone-anchored suspension technique (BAST) for lateral wall collapse.[56] They performed a randomized, prospective study and compared 6 BAST and 7 RFITT patients and their outcomes. The RFITT group had significant improvement in their NOSE and VAS scores as well as reduced collapse based on physician assessment. These improvements appear to be better than the BAST group, however the sample size was small in their study. Despite the small sample size, RFITT is a technique that warrants further evaluation.

\section{Discussion/EBM}

While much has been written with regards to nasal valve collapse and its various treatment options, there is a paucity of level I data. As mentioned by others[57, 58], there is an inherent ethical dilemma in randomizing patients to certain surgical treatments. As a result, much of our knowledge in nasal valve surgery is based on retrospective reports. That being said, both Rhee et al[59] and Spielmann et al[10] found that despite the lack of level I data, there is good 
evidence that nasal valve surgery is beneficial for patients. In 2010, Rhee et al[6] proposed guidelines for treatment of nasal valve collapse based on available literature and expert consensus. They recommended that nasal obstruction secondary to nasal valve collapse should be treated. Further studies by Lindsay[21], Rhee et al[22], and Manickavasagam et al[60] continue to show benefit based on validated assessment tools.

Despite the challenges of utilizing objective measures in nasal valve collapse[6, 17], the search for useful objective tests is ongoing. Tsounis et al[18] found good correlation between NPIF, VAS and NOSE scores. They also found that cross-sectional area and nasal airway resistance did not change significantly, therefore concluding that successful treatment of dynamic valve collapse hinged on providing rigidity to the lateral nasal wall rather than augmentation of the size of the airway. While a multitude of subjective reports have acknowledged the benefits of nasal valve surgery, objective testing can provide further insight into the parameters affected by treatment and thus help clinicians better tailor treatment algorithms.

\section{Conclusion}

Our knowledge of nasal valve collapse and outcomes of functional rhinoplasty continues to evolve. Established techniques are re-visited and validated with validated assessment tools and objective testing. New techniques are developed as our understanding of the pathophysiology increases. Despite the lack of truly randomized trials, the evidence is strong in support of functional rhinoplasty for the treatment of nasal valve collapse. Future studies are warranted to incorporate validated tools and objective measures to help standardize communication between clinicians and facilitate pooling of data. 


\section{Main Points}

- Nasal valve collapse is a significant contributor to nasal airway obstruction and should be treated.

- Despite the lack of randomized, prospective trials, there is good evidence that addressing nasal valve collapse improves the quality of life for patients.

- The technique employed for an individual patient should be based on the patient’s specific problem with the nasal valve. Each method may be suited to best fix a different aspect of nasal valve collapse..

- Future studies are warranted to incorporate validated tools and objective measures to help standardize communication between clinicians and facilitate pooling of data.

\section{Acknowledgements}

None

\section{Financial support and sponsorship}

None

\section{Conflicts of Interest}

None 


\section{References}

1. Shemirani NL, Rhee JS, Chiu AM. Nasal airway obstruction: Allergy and otolaryngology perspectives. Ann Allergy Asthma Immunol. 2008;101:593-598.

2. Aksoy F, Veyseller B, Yildirim YS et al. Role of nasal muscles in nasal valve collapse. Otolaryngol Head Neck Surg. 2010;142:365-369.

3. Toriumi DM, Josen J, Weinberger M, Tardy MEJ. Use of alar batten grafts for correction of nasal valve collapse. Arch Otolaryngol Head Neck Surg. 1997;123:802-808.

4. Schlosser RJ, Park SS. Functional nasal surgery. Otolaryngol Clin North Am. 1999;32:3751.

5. Wittkopf M, Wittkopf J, Ries WR. The diagnosis and treatment of nasal valve collapse. Curr Opin Otolaryngol Head Neck Surg. 2008;16:10-13.

6. Rhee JS, Weaver EM, Park SS et al. Clinical consensus statement: Diagnosis and management of nasal valve compromise. Otolaryngol Head Neck Surg. 2010;143:48-59.

7. Yarlagadda BB, Dolan RW. Nasal valve dysfunction: Diagnosis and treatment. Curr Opin Otolaryngol Head Neck Surg. 2011;19:25-29.

8. Bloching MB. Disorders of the nasal valve area. GMS Curr Top Otorhinolaryngol Head Neck Surg. 2007;6:Doc07.

9. Poirrier AL, Ahluwalia S, Kwame I et al. External nasal valve collapse: validation of novel outcome measurement tool. Rhinology. 2014;52:127-132.

10. Spielmann PM, White PS, Hussain SS. Surgical techniques for the treatment of nasal valve collapse: A systematic review. Laryngoscope. 2009;119:1281-1290.

11. Lanfranchi PV, Steiger J, Sparano A et al. Diagnostic and surgical endoscopy in functional septorhinoplasty. Facial Plast Surg. 2004;20:207-215. 
12. Chaves C, de Andrade CR, Ibiapina C. Objective measures for functional diagnostic of the upper airways: Practical aspects. Rhinology. 2014;52:99-103.

13. Kim DW, Rodriguez-Bruno K. Functional rhinoplasty. Facial Plast Surg Clin North Am. 2009;17:115-31, vii.

14. Edizer DT, Erisir F, Alimoglu Y, Gokce S. Nasal obstruction following septorhinoplasty: How well does acoustic rhinometry work? Eur Arch Otorhinolaryngol. 2013;270:609-613.

15. Chin D, Marcells G, Malek J et al. Nasal peak inspiratory flow (npif) as a diagnostic tool for differentiating decongestable from structural nasal obstruction. Rhinology. 2014;52:116-121.

16. Palesy T, Pratt E, Mrad N et al. Airflow and Patient-Perceived Improvement Following Rhinoplastic Correction of External Nasal Valve Dysfunction. JAMA Facial Plast Surg. $2015^{* *}$

The authors objectively measured changes in the nasal valve area using NPIF and correlated it with subjective improvements based on the NOSE scale. They also showed that improving support of the lateral nasal wall is important in external nasal valve repair.

17. Ishii LE, Rhee JS. Are diagnostic tests useful for nasal valve compromise? Laryngoscope. 2013;123:7-8.

18. Tsounis M, Swart KM, Georgalas C et al. The clinical value of peak nasal inspiratory flow, peak oral inspiratory flow, and the nasal patency index. Laryngoscope. 2014;124:26652669.*

The authors compared several objective measurements tools against the NOSE scale to evaluate for a correlation. NPIF was found to be a reliable method for assessing nasal patency. 
19. Menger DJ, Swart KM, Nolst Trenite GJ et al. Surgery of the external nasal valve: the correlation between subjective and objective measurements. Clin Otolaryngol. 2014;39:150-155.

20. Most SP. Analysis of outcomes after functional rhinoplasty using a disease-specific quality-of-life instrument. Arch Facial Plast Surg. 2006;8:306-309.

21. Lindsay RW. Disease-specific quality of life outcomes in functional rhinoplasty. Laryngoscope. 2012;122:1480-1488.

22. Rhee JS, Sullivan CD, Frank DO et al. A systematic review of patient-reported nasal obstruction scores: defining normative and symptomatic ranges in surgical patients. JAMA Facial Plast Surg. 2014;16:219-25*

The authors perform a systematic review of studies utilizing the NOSE and VAS scores to establish normative values.

23. Stewart MG, Witsell DL, Smith TL et al. Development and validation of the Nasal Obstruction Symptom Evaluation (NOSE) scale. Otolaryngol Head Neck Surg. 2004;130:157-163.

24. Bloom JD, Sridharan S, Hagiwara M et al. Reformatted computed tomography to assess the internal nasal valve and association with physical examination. Arch Facial Plast Surg. 2012;14:331-335.

25. Cho GS, Kim JH, Jang YJ. Correlation of nasal obstruction with nasal cross-sectional area measured by computed tomography in patients with nasal septal deviation. Ann Otol Rhinol Laryngol. 2012;121:239-245.

26. Moche JA, Cohen JC, Pearlman SJ. Axial computed tomography evaluation of the internal nasal valve correlates with clinical valve narrowing and patient complaint. Int Forum 
Allergy Rhinol. 2013;3:592-597.

27. Tardy ME, Garner ET. Inspiratory nasal obstruction secondary to alar and nasal valve collapse: Technique for repair using autogenous cartilage. Operative Techniques in Otolaryngology-Head and Neck Surgery. 1990;1:215-217.

28. Mendelsohn MS, Golchin K. Alar expansion and reinforcement: A new technique to manage nasal valve collapse. Arch Facial Plast Surg. 2006;8:293-299.

29. Sufyan A, Ziebarth M, Crousore N et al. Nasal batten grafts: Are patients satisfied? Arch Facial Plast Surg. 2012;14:14-19.

30. Chua DY, Park SS. Alar batten grafts. JAMA Facial Plast Surg. 2014;16:377-378.

31. Cervelli V, Spallone D, Bottini JD et al. Alar batten cartilage graft: Treatment of internal and external nasal valve collapse. Aesthetic Plast Surg. 2009;33:625-634.

32. Sufyan AS, Hrisomalos E, Kokoska MS, Shipchandler TZ. The effects of alar batten grafts on nasal airway obstruction and nasal steroid use in patients with nasal valve collapse and nasal allergic symptoms: A prospective study. JAMA Facial Plast Surg. 2013;15:182-186. ** The authors showed that by repairing nasal valve collapse, nasal allergic symptoms can be improved and also reduce the use of nasal steroids.

33. Khosh MM, Jen A, Honrado C, Pearlman SJ. Nasal valve reconstruction: Experience in 53 consecutive patients. Arch Facial Plast Surg. 2004;6:167-171.

34. Millman B. Alar batten grafting for management of the collapsed nasal valve. Laryngoscope. 2002;112:574-579.

35. Sheen JH. Spreader graft: A method of reconstructing the roof of the middle nasal vault following rhinoplasty. Plast Reconstr Surg. 1984;73:230-239.

36. Reiffel AJ, Cross KJ, Spinelli HM. Nasal spreader grafts: a comparison of medpor to 
autologous tissue reconstruction. Ann Plast Surg. 2011;66:24-28.

37. Teymoortash A, Fasunla JA, Sazgar AA. The value of spreader grafts in rhinoplasty: a critical review. Eur Arch Otorhinolaryngol. 2012;269:1411-1416.

38. Yoo DB, Jen A. Endonasal placement of spreader grafts: experience in 41 consecutive patients. Arch Facial Plast Surg. 2012;14:318-322.

39. Saedi B, Amaly A, Gharavis V et al. Spreader flaps do not change early functional outcomes in reduction rhinoplasty: A randomized control trial. Am J Rhinol Allergy. 2014;28:70-74.

40. de Pochat VD, Alonso N, Mendes RR et al. Nasal patency after open rhinoplasty with spreader grafts. J Plast Reconstr Aesthet Surg. 2012;65:732-738.

41. Jalali MM. Comparison of effects of spreader grafts and flaring sutures on nasal airway resistance in rhinoplasty. Eur Arch Otorhinolaryngol. 2014

42. Shadfar S, Shockley WW, Fleischman GM et al. Characterization of postoperative changes in nasal airflow using a cadaveric computational fluid dynamics model: Supporting the internal nasal valve. JAMA Facial Plast Surg. 2014;16:319-327.

43. Oneal RM, Berkowitz RL. Upper lateral cartilage spreader flaps in rhinoplasty. Aesthet Surg J. 1998;18:370-371.

44. Yoo S, Most SP. Nasal airway preservation using the autospreader technique: analysis of outcomes using a disease-specific quality-of-life instrument. Arch Facial Plast Surg. 2011;13:231-233.

45. Park SS. The flaring suture to augment the repair of the dysfunctional nasal valve. Plast Reconstr Surg. 1998;101:1120-1122.

46. Schlosser RJ, Park SS. Surgery for the dysfunctional nasal valve. Cadaveric analysis and 
clinical outcomes. Arch Facial Plast Surg. 1999;1:105-110.

47. Troell RJ, Powell NB, Riley RW, Li KK. Evaluation of a new procedure for nasal alar rim and valve collapse: nasal alar rim reconstruction. Otolaryngol Head Neck Surg. 2000;122:204-211.

48. Guyuron B. Alar rim deformities. Plast Reconstr Surg. 2001;107:856-863.

49. Rohrich RJ, Raniere JJ, Ha RY. The alar contour graft: correction and prevention of alar rim deformities in rhinoplasty. Plast Reconstr Surg. 2002;109:2495-505; discussion 2506.

50. Boahene KD, Hilger PA. Alar rim grafting in rhinoplasty: Indications, technique, and outcomes. Arch Facial Plast Surg. 2009;11:285-289.

51. Clark JM, Cook TA. The 'butterfly’ graft in functional secondary rhinoplasty. Laryngoscope. 2002;112:1917-1925.

52. Friedman O, Cook TA. Conchal cartilage butterfly graft in primary functional rhinoplasty. Laryngoscope. 2009;119:255-262.

53. Stacey DH, Cook TA, Marcus BC. Correction of internal nasal valve stenosis: A single surgeon comparison of butterfly versus traditional spreader grafts. Ann Plast Surg. 2009;63:280-284.

54. Chaiet SR, Marcus BC. Nasal tip volume analysis after butterfly graft. Ann Plast Surg. 2014;72:9-12.

55. Seren E. A new surgical method of dynamic nasal valve collapse. Arch Otolaryngol Head Neck Surg. 2009;135:1010-1014.

56. Weissman JD, Most SP. Radiofrequency Thermotherapy vs Bone-Anchored Suspension for Treatment of Lateral Nasal Wall Insufficiency: A Randomized Clinical Trial. JAMA Facial Plast Surg. $2015 *$ 
The authors propose a novel method of treating nasal valve collapse and utilize a validated assessment tool to evaluate patient outcomes.

57. Cannon DE, Rhee JS. Evidence-based practice: Functional rhinoplasty. Otolaryngol Clin North Am. 2012;45:1033-1043.

58. Angelos PC, Been MJ, Toriumi DM. Contemporary review of rhinoplasty. Arch Facial Plast Surg. 2012;14:238-247.

59. Rhee JS, Arganbright JM, McMullin BT, Hannley M. Evidence supporting functional rhinoplasty or nasal valve repair: A 25-year systematic review. Otolaryngol Head Neck Surg. 2008;139:10-20.

60. Manickavasagam J, Wong S, Varabei V, Raghavan U. Nasal valve surgery: Assessment of quality of life with the glasgow benefit inventory. Ear Nose Throat J. 2014;93:174-6, 180. 